\title{
Research of Smart Car's Speed Control Based on the Internal Model Control
}

\author{
Han Yu, ${ }^{1}$ Hamid Reza Karimi, ${ }^{2}$ and Xuemei $\mathrm{Zhu}^{1}$ \\ ${ }^{1}$ Nanjing Normal University, Jiangsu 210042, China \\ ${ }^{2}$ Department of Engineering, Faculty of Engineering and Science, The University of Agder, 4898 Grimstad, Norway
}

Correspondence should be addressed to Han Yu; 466313381@qq.com

Received 4 April 2014; Revised 16 May 2014; Accepted 18 May 2014; Published 4 June 2014

Academic Editor: Shen Yin

Copyright (C) 2014 Han Yu et al. This is an open access article distributed under the Creative Commons Attribution License, which permits unrestricted use, distribution, and reproduction in any medium, provided the original work is properly cited.

Based on analyzing principle about the smart car's speed control system, the system mathematical model is built. Considering the control optimization, a novel control scheme is proposed based on internal model control, and the internal model controller of speed control system is established. Regarding this subject, the internal control theory is introduced to verify the control performance; the traditional PID control method is employed in the experiment. The experiment indicates that the proposed method based on internal model control is easier to determine parameter and has a well robust and good control result of smart car's speed.

\section{Introduction}

Cars have been the most important transportation for the progress of science and technology. While we have to face the more serious traffic problems meanwhile enjoying the convenience of the cars, the intelligent transportation system (ITS), which was put forward in recent years, has made people believe that the problems would be solved. However, due to the restriction of experimental environment and cost, research about ITS is very hard to open up in real life. Scaling down the model of ITS in proportional could get a high accuracy simulation and analysis of the real one [1-3]. Therefore, a kind of model called smart car system [4-6] has gotten more and more attention in the study of ITS. The study core is the control scheme of smart cars; as the control process of smart cars could reflect basis characters and all keys of ITS, it could effectively support the research and technology on related fields. Therefore the study about smart cars has obtained a great practical significance and scientific value.

The research about smart car includes the technology, methods of navigation, positioning, motion planning, and speed control. The technology and methods of the speed control are important to the achievement of smart car. There are some algorithms of control, such as an approach based on fault-tolerant control [7] and data-driven support [8-11], using fuzzy-PID mode algorithm [12] and adaptive sliding mode algorithm $[13,14]$. Among all of the algorithms, PID controller $[15,16]$ has been widely used in ordinary vehicle cruise control because its structure is simple and the effect is significant. However, the parameter adjustment of PID controller is complex, and its control effect is not ideal for the time delay systems. For this, we put forward a novel control scheme of speed control based on internal model control (IMC); we find out that internal model control has more and more applications in the control of multivariable time delay process for its simple structure, convenient parameter setting, good robustness, and some compensation effect of the time delay process $[17,18]$. This paper sets up a mathematical model of speed control of smart cars system, which consists of navigation detection module, drive control module, speed detection module, system debugging module, power module, and other components based on the core of Freescale's 32bit microprocessor MK60N512VMD100, researched on the speed control of smart cars system and proposed a control strategy based on IMC, made a study of control effect compared with the PID controller. The result shows that, the strategy of IMC for speed control could attain, even better than, the result of PID control, greatly reduce the difficulty of adjusting the parameter of controller, and has strong value in application. 


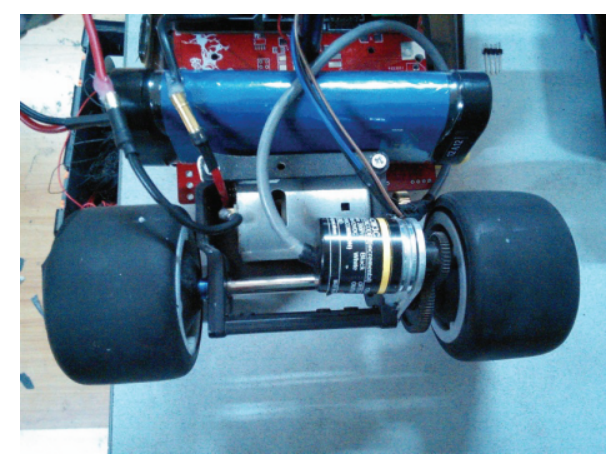

Figure 1: Model of smart car.

\section{Introduction of Speed Control System}

Smart car control system is a closed loop control system, which consisted of speed detection module, DC motor driver module, and power supply module. The error between set speed and measurement speed is the input of speed controller, by which the DC motor driver attains the corresponding driving signal; thus the smart car can be driven by a certain speed.

It is hard to build an accurate mathematical model through the analysis of the car's physical characteristics with the reason that not only the motor type, tire size, and the weight of the car are uncertain but also the power voltage, the wind resistance, and the road friction keep changing. With the analysis of the large amount of experimental data and the reference of the related paper $[19,20]$, it is found that one-order inertial lag model can be used, which is defined as follows:

$$
G(s)=\frac{K}{1+T s} e^{-\tau s}
$$

It is well known that the measured data of the encoder of the smart car has a linear relationship to the real speed of the smart car through some practical testing; therefore the real speed of the smart car can be easily gained and expressed by the encoder. The smart car model with four wheels is shown in Figure 1, which is driven by a single DC motor, and its length and width are $35 \mathrm{~cm}$ and $18 \mathrm{~cm}$, respectively.

The relationship between the data of encoder and the time is shown in Figure 2 when the battery voltage is $7.5 \mathrm{~V}$.

A specific mathematical model can be achieved by the processing and the analysis to the measured data, and the parameters of model are as follows: $K=1, T=200$, and $\tau=0.5$. In this paper, several comparative experiments will be done based on the above mathematical model of the speed control system of the smart car, which is as follows:

$$
G(s)=\frac{1}{1+200 s} e^{-0.5 s}
$$

\section{Basic Principle of the Inner Model Control}

In order to have a good control result and easy operation to the speed control system, we proposed a control scheme based on IMC after the analysis of the mathematic model.

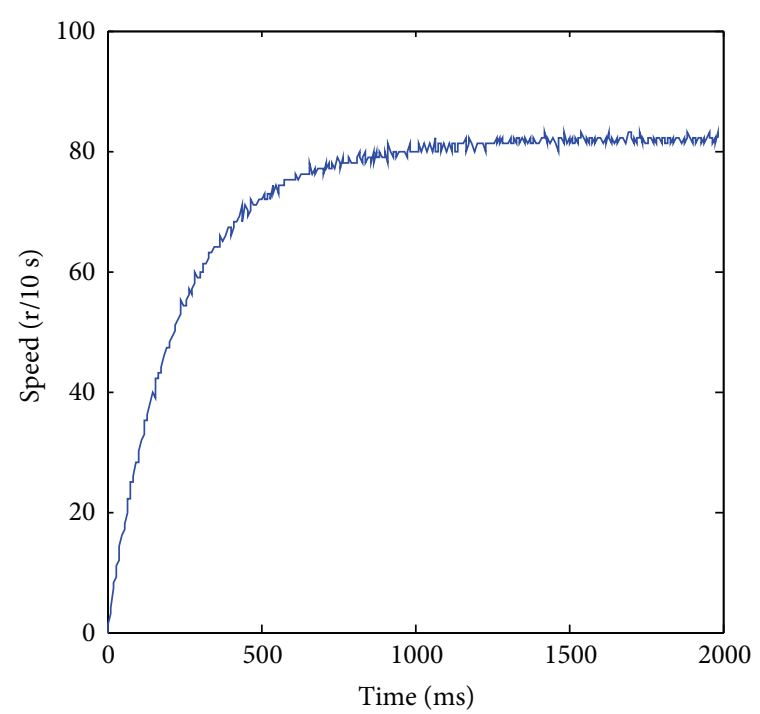

Figure 2: Relationship between the car's speed and time.

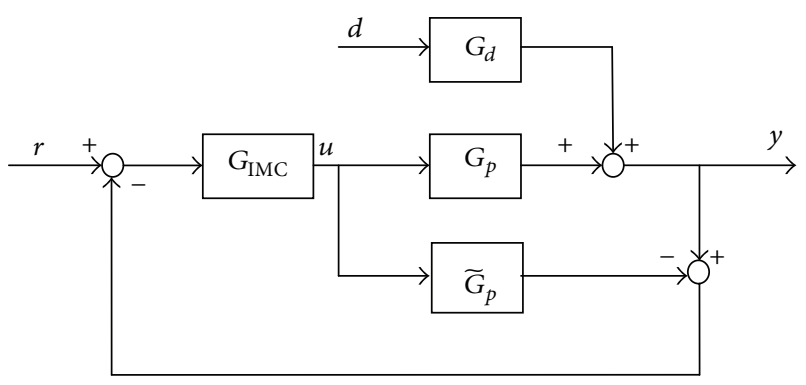

Figure 3: The IMC structure diagram.

The IMC is a kind of control strategy whose controller is designed on the basis of the process mathematical model. The IMC strategy was firstly proposed by Garcia and Morari in 1982, which caught the researchers and the engineers' attention simultaneously for its merits of simplicity, good tracking performance, robustness, and ability of eliminating the unmeasured disturbance. It does not need accurate object model and can probably acquire the better robustness after introducing the filter. In addition, the inner model controller not only is able to adjust parameters easily but also has a dual stability and ideal controller features of zero steady-state error.

The inner control model structure is shown in Figure 3, where $G_{\text {IMC }}$ represents the transfer function of the controller, $G_{P}$ represents the process, $\widetilde{G}_{P}$ represents the process model, and $G_{d}$ represents the transfer function of the disturbance. In order to gain the two transfer functions between the input and the output (one is the input $r$ and the output $y$ and the other is the input $d$ and the output $y$ ) in Figure 3, the structure in Figure 3 can be converted into the equivalent IMC structure which is shown in Figure 4; thus it is convenient to obtain the 


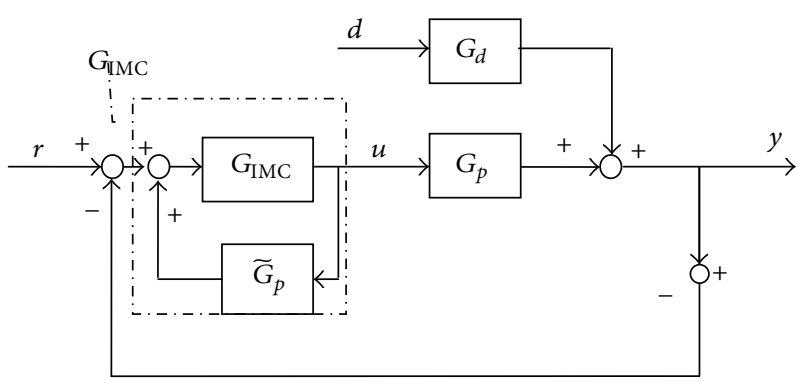

FIGURE 4: The equivalent IMC structure.

transfer function of the inner feedback controller, which is defined as follows:

$$
G_{C}(s)=\frac{G_{\mathrm{IMC}}(s)}{1-G_{\mathrm{IMC}}(s) \widetilde{G}_{P}(s)} .
$$

The transfer functions of the input and the output are defined as

$$
\begin{aligned}
& \frac{y(s)}{r(s)}=\frac{G_{\mathrm{IMC}}(s) G_{P}(s)}{1+G_{\mathrm{IMC}}(s)\left[G_{P}(s)-\widetilde{G}_{P}(s)\right]}, \\
& \frac{y(s)}{d(s)}=\frac{\left[1-G_{\mathrm{IMC}}(s) \widetilde{G}_{P}(s)\right] G_{d}(s)}{1+G_{\mathrm{IMC}}(s)\left[G_{P}(s)-\widetilde{G}_{P}(s)\right]} .
\end{aligned}
$$

The feedback signal of the system is defined as

$$
\tilde{d}(s)=\left[G_{P}(s)-\widetilde{G}_{P}(s)\right] u(s)+G_{d}(s) d(s) .
$$

The input of the model is equal to the process output if the above model is accurate (that is to say that expression $G_{P}(s)=$ $\widetilde{G}_{P}(s)$ is established) and has no external disturbances, so the value of the feedback signal is zero. But, overcoming the disturbance is one of the most important tasks in every process control and it is difficult to avoid the uncertainty of the model; therefore the feedback signal $\widetilde{d}(s)$ reflects the influence of the system on the uncertainty of the process model and the disturbance.

\section{Design and Application of the Inner Model Controller}

Due to the control scheme we proposed IMC which has simple operation and good tracking performance; we began to design an inner model controller for the speed control system to validate it.

For one-order inertial lag model, the design of the inner model controller is divided into two parts including the design of ideal controller and the introduction of the filter.

The IMC structure in Figures 3 and 4 can be transferred into the structure in Figure 5. The process model $\widetilde{G}_{P}$ is decomposed into the product of two parts, which is as follows:

$$
\widetilde{G}_{P}(s)=\widetilde{G}_{P+}(s) \cdot \widetilde{G}_{P-}(s),
$$

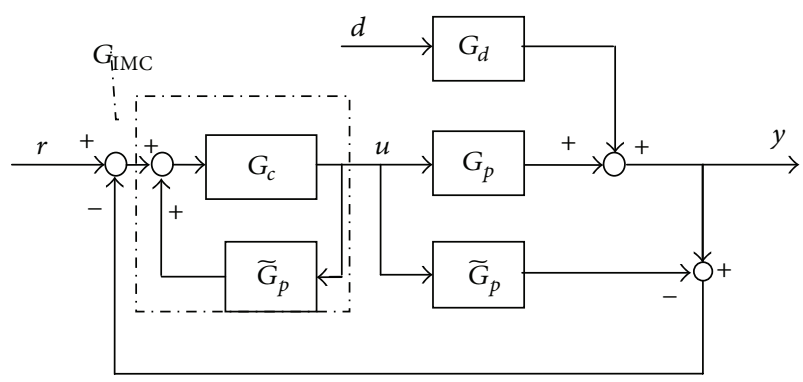

FIGURE 5: Relationship between the IMC structure and the feedback control structure.

where $\widetilde{G}_{P-}(s)$ is a transfer function of the full-pass filter containing all the lag and RHP zero and it is also with characteristics of a minimum phase.

The defined IMC controller is as follows:

$$
\begin{aligned}
G_{\mathrm{IMC}}(s) & =\widetilde{G}_{p^{-}}^{-1}(s) f(s), \\
f(s) & =\frac{1}{(1+a s)^{n}},
\end{aligned}
$$

where $f(s)$ is a low pass filter and the parameter $a$ is the only one parameter needed to be designed, and the following inequality should be met up to satisfy the requirement of the robustness which is defined as

$$
\frac{G_{\text {IMC }}(\infty)}{G_{\text {IMC }}(0)} \leq 20 .
$$

It is known that the speed control model in the smart car is one-order inertial lag model from the expression (2); the proper results are as follows by computing $n=1, a \geq 10$, and $\widetilde{G}_{p-}^{-1}(s)=1+200 s$. The concrete transfer function of the inner model controller is as follows:

$$
G_{\mathrm{IMC}}(s)=\frac{1+200 s}{1+a s} \quad(a \geq 10) .
$$

\section{Simulation and Analysis of the Results}

This paper mainly discusses the design of the speed controller of the smart car. However, due to the difficulty of building the speed control model of the smart car and the influence of the battery voltage, the environment friction coefficient, and so on, the author constructs an approximate one-order inertia pure lag mathematical model aiming at the speed control system with four wheels and one single DC motor, and the parameter of the motor model is gained by a large number of experiments. The mathematical model of the motor is displayed in expression (2).

As is said above, the mathematical model of the inner model controller is displayed in expression (9). Expression (2) combined with expression (9) can get the inner model controller of this smart car's speed control system; the transfer function of the inner model controller is as follows:

$$
G_{\mathrm{IMC}}(s)=\frac{1+200 s}{1+10 s} .
$$




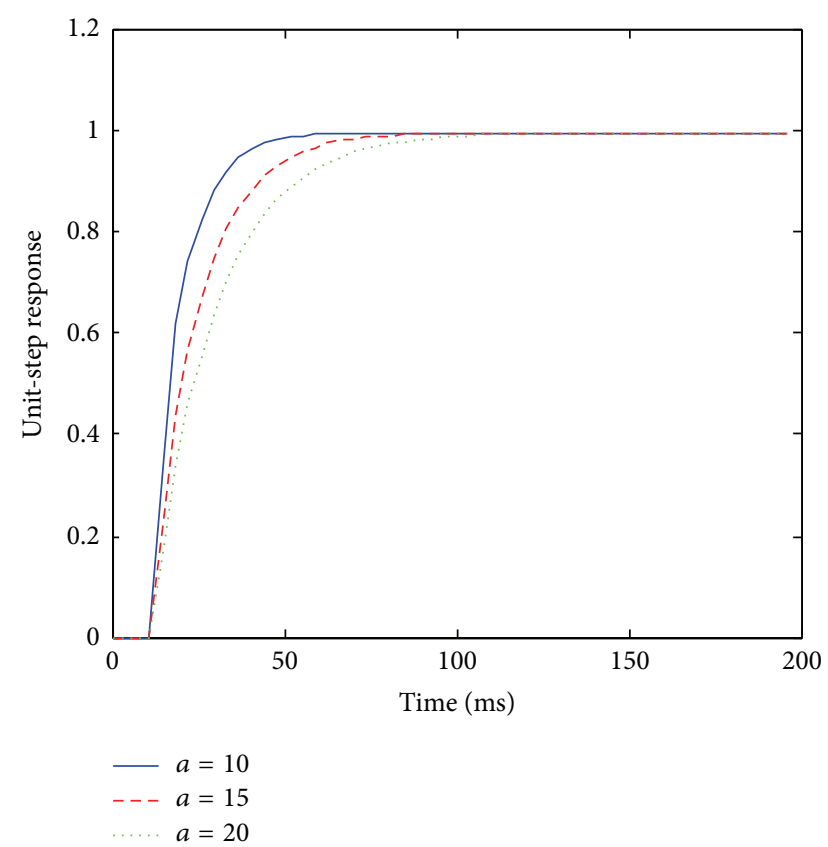

FIGURE 6: Different output results of the IMC controller.

As is discussed above, it is easy to find that the IMC controller has only one parameter about the filter coefficients in the case of the known system. The only one parameter makes it easier to reach the expected result. When looking for the best parameter value, the strategy based on IMC could not be affected by other parameters more directly and conveniently.

The different results are shown in Figure 6 by keeping the same step input and changing the parameter $a$.

Figure 6 shows that the control system has a good and stable control effect when $a=10, a=15$, or $a=20$, so it is inferred that the value of the parameter $a$ can be chosen from a certain range under the premise of the good control performance. One conclusion from the above analysis is that the inner model controller has good robustness, while the most smart car's speed control system accepts the PID controller. The good robustness of the IMC brings a large range of parameter adjustments and makes it easier to obtain a good control effect.

In this paper, aiming at the smart car's speed control system with characteristics of first-order inertia, the author has simulated the effect of the IMC controller and the PID controller. According to the actual debugging, one group of good parameters of the PID controller is as follows: $K_{p}=25$, $T_{i}=5$, and $T_{d}=0.25$, so the transfer function of the PID controller is employed as follows:

$$
G_{C}(s)=25\left(1+0.2 \frac{1}{s}+0.25 s\right) \text {. }
$$

The simulation result is in Figure 7 using the IMC controller and the PID controller to control the smart car's speed, respectively, when the input is the step signal given.

According to the analysis of Figure 7, the PID controller has some overshoot and its settling time is longer while the

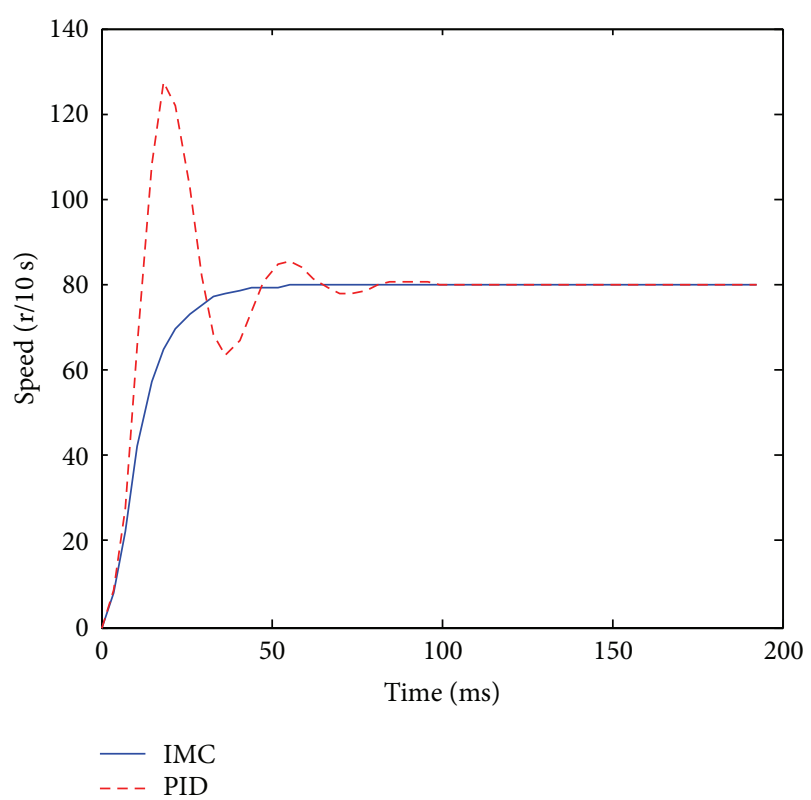

FIgure 7: Comparison to the effect of the IMC and the PID controller with step input.

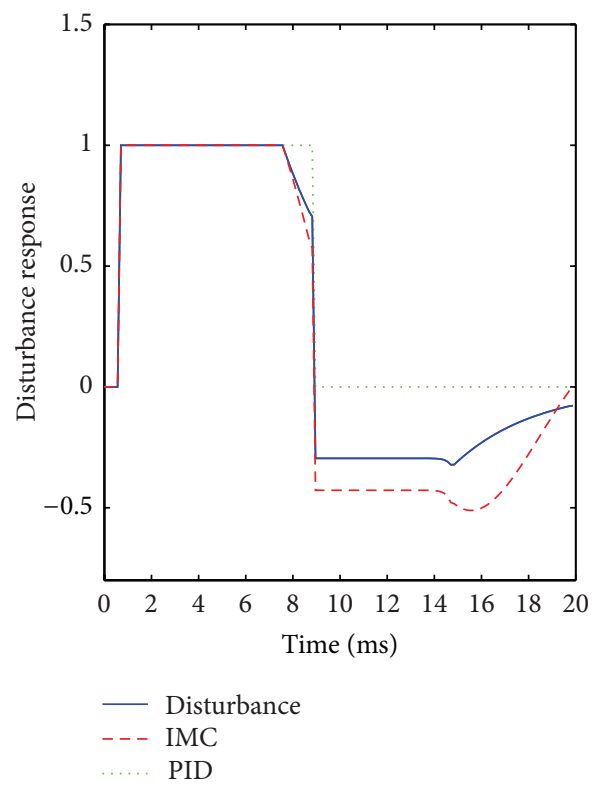

FIGURE 8: Effects of the IMC and the PID controller with disturbance input.

IMC controller has less settling time with no steady-state error; hence the IMC controller has more excellent stability and rapidity than the PID controller. Therefore, the strategy based on IMC can be faster and more accurate to achieve the desired control requirements.

The simulation result is in Figure 8 using the IMC controller and the PID controller to control the smart car's speed, respectively, with disturbance input.

According to the analysis of Figure 8, the IMC controller is less affected by the disturbance than the PID controller 
in the same disturbance input; therefore the IMC controller has better capacity of resisting disturbance than the PID controller. The noise immunity of IMC makes it get a better control in disturbance environments instead of deviating from the expected requirements.

\section{Conclusion}

In this paper, we propose a strategy of smart car's speed control system which is based on the IMC and do the research and analysis of its simulation. We can conclude that the system based on IMC has a more superior stability, rapidity, and capacity of resisting disturbance than the one based on the traditional PID control from the simulation results of the two strategies. Meanwhile, the simulation of IMC controller in different parameters within a certain range proves that the IMC has a good ability to adapt. In conclusion, the speed control based on IMC could attain the result of PID control. Moreover, the strategy of IMC could greatly reduce the difficulty of adjusting the parameter of controller. In application, the scheme based on the IMC makes it easier to reach the same control effect compared with other strategies and has strong operability and less demand on the environment. Therefore, our scheme which was proposed based on the IMC can be good for the smart car's speed control system. With the rapid development of ITS, how to create a precise speed control model in order to achieve a more precise speed control is the next step to in-depth study of the subject.

\section{Conflict of Interests}

The authors declare that there is no conflict of interests regarding the publication of this paper.

\section{Acknowledgments}

This present work was supported partially by the PolishNorwegian Research Programme (Project no. Pol-Nor/ 200957/47/2013). The authors highly appreciate the above financial support.

\section{References}

[1] C. Sommer, R. German, and F. Dressler, "Bidirectionally coupled network and road traffic simulation for improved IVC analysis," IEEE Transactions on Mobile Computing, vol. 10, no. 1, pp. 3-15, 2011.

[2] G. Min and S. Yagang, "Traffic simulation and forecasting system in Beijing," Engineering Sciences, vol. 8, no. 1, pp. 49-52, 2010.

[3] L. Li, Y.-A. Liu, and B.-H. Tang, "SNMS: an intelligent transportation system network architecture based on WSN and P2P network," Journal of China Universities of Posts and Telecommunications, vol. 14, no. 1, pp. 65-70, 2007.

[4] J. Sun, Z.-H. Wu, and G. Pan, "Context-aware smart car: from model to prototype," Journal of Zhejiang University: Science A, vol. 10, no. 7, pp. 1049-1059, 2009.
[5] Z. Wu, Y. Liu, and G. Pan, "A smart car control model for brake comfort based on car following," IEEE Transactions on Intelligent Transportation Systems, vol. 10, no. 1, pp. 42-46, 2009.

[6] X. Wang, H. Li, and Y. Zhu, "Design on automatic control system for smart car based on $\mu \mathrm{C} / \mathrm{OS}$-II," in Proceedings of the 2012 IEEE 2nd International Conference on Cloud Computing and Intelligence Systems (CCIS '12), vol. 3, pp. 1188-1191, 2012.

[7] S. Yin, H. Luo, and S. Ding, "Real-time implementation of faulttolerant control systems with performance optimization," IEEE Transactions on Industrial Electronics, vol. 64, no. 5, pp. 24022411, 2014.

[8] S. Yin, G. Wang, and H. R. Karimi, "Data-driven design of robust fault detection system for wind turbines," Mechatronics, vol. 24, no. 4, pp. 298-306, 2014.

[9] S. Yin, S. X. Ding, A. H. Abandan Sari, and H. Hao, "Data-driven monitoring for stochastic systems and its application on batch process," International Journal of Systems Science, vol. 44, no. 7, pp. 1366-1376, 2013.

[10] S. Yin, S. X. Ding, A. Haghani, H. Hao, and P. Zhang, "A comparison study of basic data-driven fault diagnosis and process monitoring methods on the benchmark Tennessee Eastman process," Journal of Process Control, vol. 22, no. 9, pp. 1567-1581, 2012.

[11] S. Yin, X. Yang, and H. R. Karimi, "Data-driven adaptive observer for fault diagnosis," Mathematical Problems in Engineering, vol. 2012, Article ID 832836, 21 pages, 2012.

[12] L. Chai, M. Gong, X. Liu, X. Zhang, and C. Hu, "Implementation of fuzzy-PID in smart car control," Journal of Measurement Science and Instrumentation, vol. 1, pp. 75-77, 2010.

[13] B. Xiao, Q. Hu, and Y. Zhang, "Adaptive sliding mode fault tolerant attitude tracking control for flexible spacecraft under actuator saturation," IEEE Transactions on Control Systems Technology, vol. 20, no. 6, pp. 1605-1612, 2012.

[14] B. Xiao, Q.-L. Hu, and G. Ma, "Adaptive sliding mode backstepping control for attitude tracking of flexible spacecraft under input saturation and singularity," Proceedings of the Institution of Mechanical Engineers, Part G: Journal of Aerospace Engineering, vol. 224, no. 2, pp. 199-214, 2010.

[15] Z. Wang and Y. Liu, "Design of smart car speed control system based on fuzzy classifying and PI speed regulation," in Proceedings of the 2010 2nd International Conference on Industrial Mechatronics and Automation (ICIMA '10), pp. 333336, May 2010.

[16] S. Zhendong, L. Ke, Y. Qingzhou, O. Lei, and Z. Xingxing, "Smart car control system based on infrared sensor," Journal of Hubei Automotive Industries Institute, vol. 3, pp. 11-14, 2007.

[17] T. Liu, F. Gao, and Y. Wang, "IMC-based iterative learning control for batch processes with uncertain time delay," Journal of Process Control, vol. 20, no. 2, pp. 173-180, 2010.

[18] S. M. Zhang, W. W. Deng, Y. Wang, and B. X. Shang, "An internal model control (IMC) algorithm for automatic trajectory tracking," Advanced Materials Research, vol. 712-715, pp. 2296-2302, 2013.

[19] Z. Wu, L. Zhao, and L. Feng, "Control of intelligent vehicle based on fractional order PID," Control Engineering of China, vol. 3, pp. 401-404, 2011.

[20] J. Lei, M. Luo, and C. Z. Chu, "Design and development of smart car DC motor speed control system," in Proceedings of the 31st Chinese Control Conference (CCC '12), pp. 4916-4919, July 2012. 


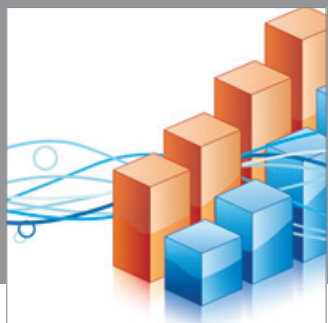

Advances in

Operations Research

mansans

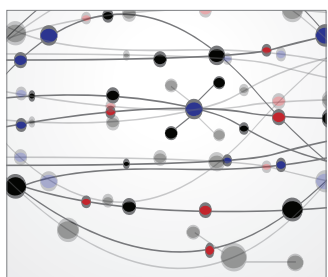

The Scientific World Journal
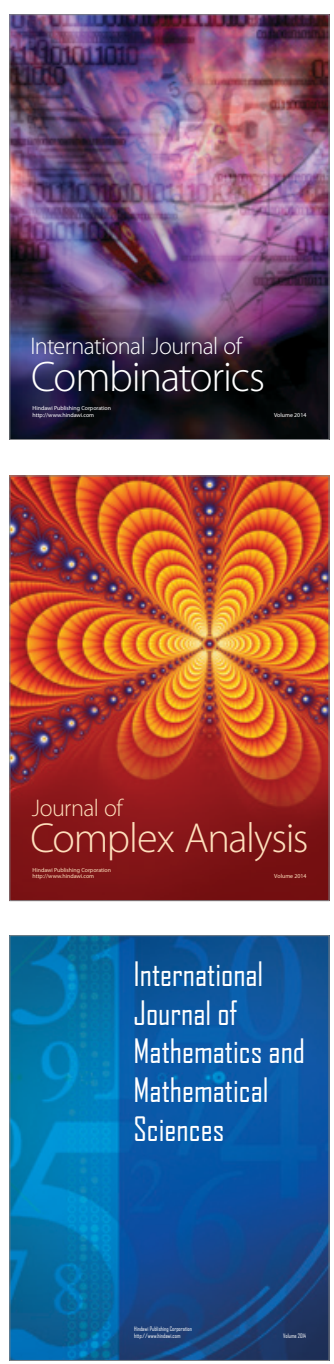
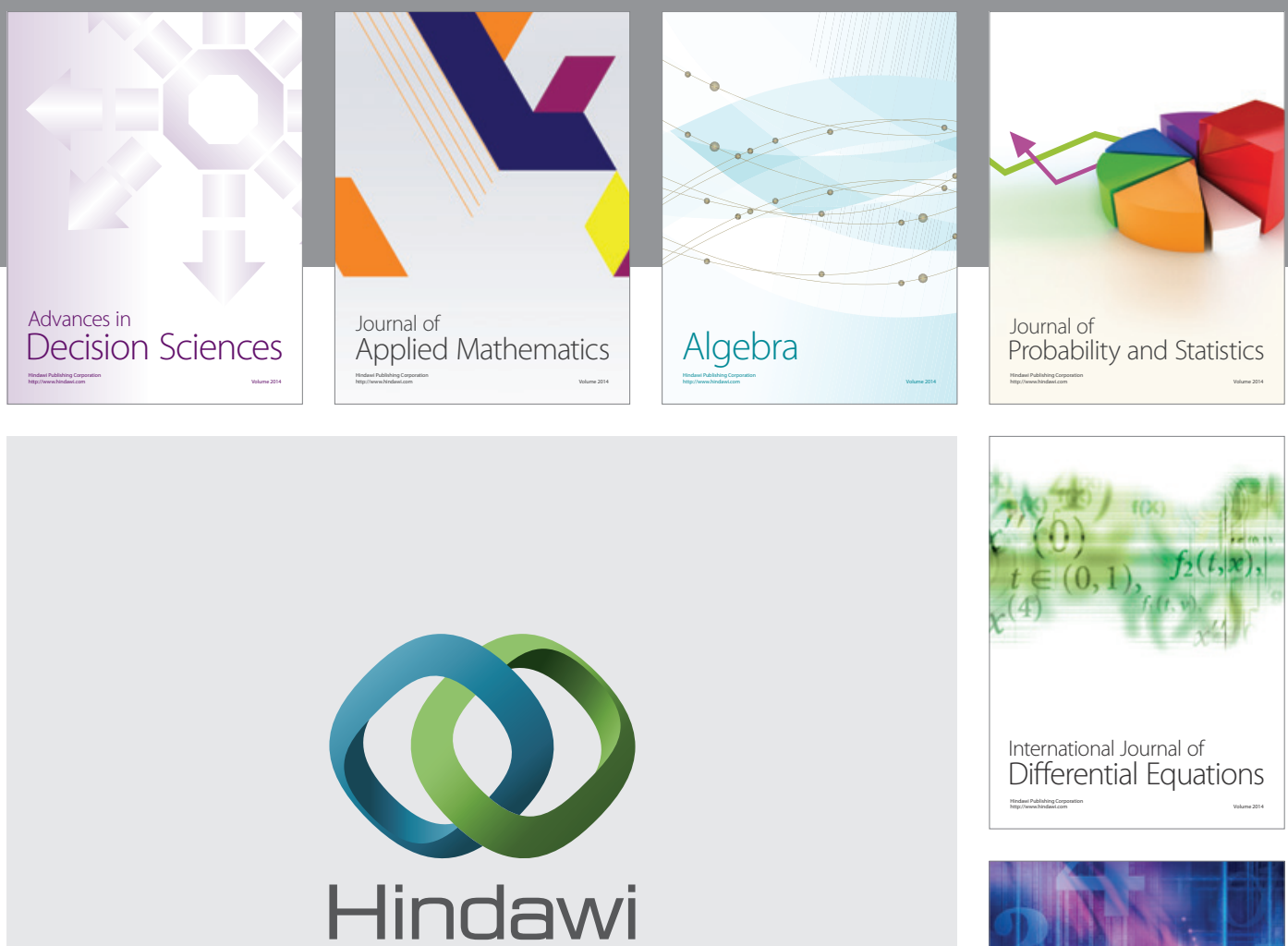

Submit your manuscripts at http://www.hindawi.com
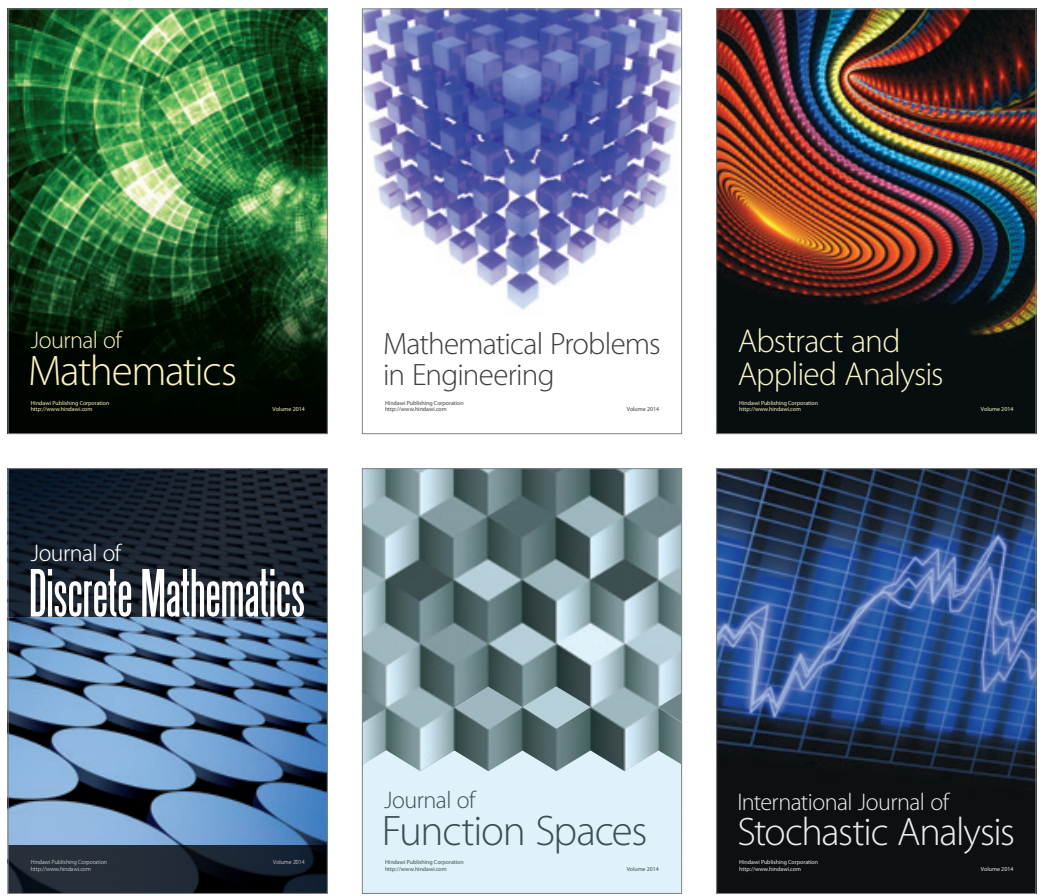

Journal of

Function Spaces

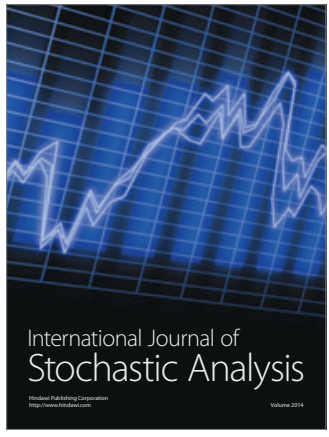

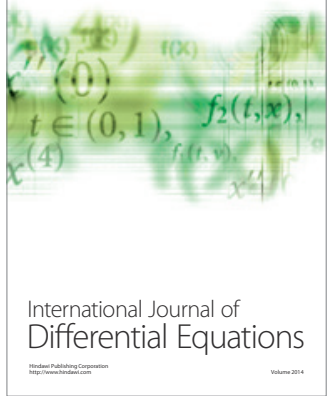
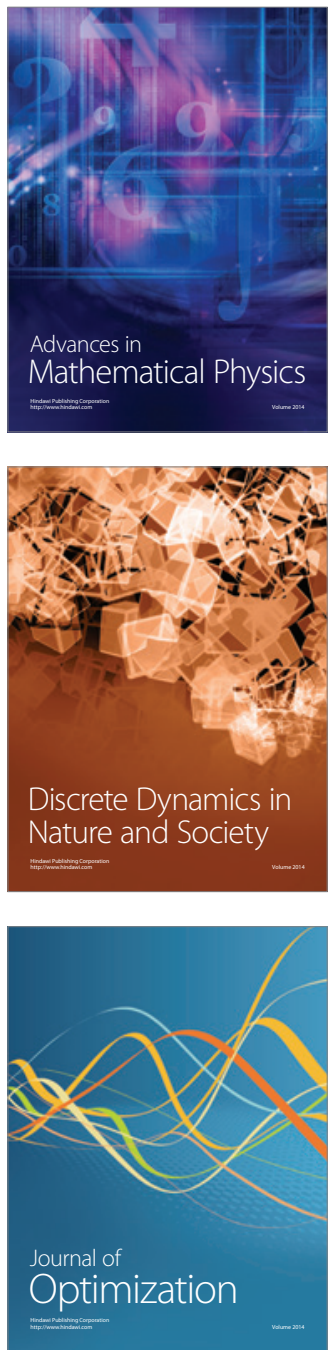